\title{
INSATISFAÇÃO NO TRABALHO DE ENFERMEIROS DE UM HOSPITAL UNIVERSITÁRIO*
}

Marisa Pires de Morais'1,Júlia Trevisan Martins², Maria Lucia do Carmo Cruz Robazzi³, Alexandrina Aparecida Maciel Cardelli²

'Enfermeira. Universidade Estadual de Londrina. Londrina-PR-Brasil.

${ }^{2}$ Enfermeira. Doutora em Enfermagem. Universidade Estadual de Londrina. Londrina-PR-Brasil.

${ }^{3}$ Enfermeira. Doutora em Enfermagem. Universidade de São Paulo. São Paulo-SP-Brasil.

RESUMO: O objetivo deste estudo foi identificar os fatores geradores de insatisfação no trabalho de enfermeiros de um hospital universitário. Pesquisa de natureza qualitativa, abordagem descritivo-exploratória, com participação de 15 enfermeiros. A coleta de dados ocorreu no período de novembro de 2011 a maio de 2012, por meio de entrevista semiestruturada e os depoimentos foram submetidas à Análise de Conteúdo. O estudo foi analisado e aprovado pelo Comitê de Ética em Pesquisa Envolvendo Seres Humanos da UEL, pelo parecer $n^{\circ}$ 257/ 2011. Como resultado obteve-se a categoria denominada de vivências de insatisfação no trabalho do enfermeiro, com as seguintes subcategorias: a falta de cooperação entre os colegas, a sobrecarga de trabalho, o não reconhecimento do trabalho realizado, a escassez de recursos humanos e materiais e o trabalho em uma instituição pública. Conclui-se que as vivências de insatisfação identificadas estão relacionadas tanto aos aspectos pessoais como institucionais.

DESCRITORES: Saúde do trabalhador; Enfermagem, Condições de trabalho.

\section{DISSATISFACTION IN THE WORK OF NURSES OF A UNIVERSITY HOSPITAL}

\begin{abstract}
This study aimed to identify the factors which cause dissatisfaction in the work of nurses in a university hospital. A total of 15 nurses participated in this qualitative study with a descriptive-exploratory approach. Data collection occurred in November 2011 - May 2012 through semi-structured interviews; the accounts were subjected to Content Analysis. The category termed Experiences of dissatisfaction in the work of the nurse was obtained as a result, with the following subcategories: the lack of co-operation between colleagues, work overload, non-recognition of the work undertaken, the scarcity of human and material resources, and the work in a public institution. It is concluded that the experiences of dissatisfaction identified are related both to personal and institutional aspects. DESCRIPTORS: Worker's health; Nursing; Work conditions.
\end{abstract}

\section{INSATISFACCIÓN EN EL TRABAJO DE ENFERMEROS DE UN HOSPITAL UNIVERSITARIO}

RESUMEN: El objetivo de este estudio fue identificar los factores que generan insatisfacción en el trabajo de enfermeros de un hospital universitario. Investigación de naturaleza cualitativa, abordaje descriptivo exploratorio, con participación de 15 enfermeros. Los datos fueron obtenidos en el periodo de noviembre de 2011 a mayo de 2012, por medio de entrevista semiestructurada y las deposiciones fueron sometidas al Análisis de Contenido. Como resultado se ha llegado a la categoría llamada de vivencias de insatisfacción en el trabajo del enfermero, con las siguientes subcategorías: falta de cooperación entre los compañeros, sobrecarga de trabajo, no reconocimiento del trabajo realizado, escasez de recursos humanos y materiales y el trabajo en una institución pública. Se concluye que las vivencias de insatisfacción identificadas están relacionadas tanto a los aspectos personales como a los institucionales. DESCRIPTORES: Salud del trabajador; Enfermería, Condiciones de trabajo.

*Artigo extraído da dissertação "Satisfação e insatisfação no trabalho de enfermeiros de um Hospital Universitário". Programa Mestrado em Enfermagem Universidade Estadual de Londrina 2011-2012.

Recebido: $14 / 03 / 2013$ Finalizado: $13 / 02 / 2014$

Autor correspondente:

Marisa Pires de Morais

Universidade Estadual de Londrina

Av. Roberto Koch, 30 - 86038-440 - Londrina-PR-Brasil

E-mail: marisapiresmorais@gmail.com 


\section{INTRODUÇÃO}

Devido o processo de globalização ocorreram transformações econômicas e sociais que alteraram a relação entre o homem e o seu labor. Tem-se observado também a busca cada vez maior por aumento da produtividade, porém, agregada a um baixo custo de produção, com a finalidade de se conseguir produtos mais competitivos no mundo capitalista. Devido essas exigências ocorre aumento do ritmo e da sobrecarga de trabalho, o que pode gerar insatisfação dos trabalhadores ao desenvolver suas atividades, interferindo, assim no seu processo saúde e doença ${ }^{(1)}$.

O trabalho ocupa lugar central na vida do ser humano e dependendo de como é o seu processo organizativo e como é executado, precisa de ações que atendam às necessidades dos trabalhadores em toda sua complexidade. Porém, nem sempre é possível atende-las, assim, sentimentos de insatisfação, de sofrimento, desinteresse, entre outros, tornam-se factíveis de acontecer $^{(2)}$.

A organização laboral exerce ação específica sobre os seres humanos cujo impacto acontece no aparelho psíquico e, de acordo com certas condições, o resultado da reciprocidade do homem com o trabalho resulta em sofrimento e insatisfação. Isso pode ocorrer devido ao choque entre a personalidade do homem, seu projeto individual e a prescrição imposta pela organização do trabalho, que não leva em consideração essas subjetividades. Entretanto, se a relação do homem com a organização das atividades é favorável, o trabalho também pode se constituir como fonte de prazer e satisfação(3).

Na concepção Dejouriana, a insatisfação no trabalho está diretamente relacionada com o conteúdo da tarefa realizada pelo trabalhador. Pode ser decorrente de sentimentos relacionados com a obrigação de realizar tarefas desinteressantes, sem significado ou, de inutilidade; por desconhecer o que representa o labor no conjunto das atividades na instituição; pela desqualificação; em função de questões salariais; na valorização do trabalho, em aspectos como responsabilidade, risco ou conhecimentos necessários ${ }^{(3)}$.

Os trabalhadores da enfermagem desenvolvem suas atividades de forma fragmentada e individual, convivem com a dor, com o sofrimento alheio, com a recuperação e o risco de morte iminente dos pacientes, entre outros fatores. Assim, eles estão sujeitos a sentimentos de insatisfação e sofrimento que podem contribuir para o desgaste mental, psíquico e físico ${ }^{(4)}$.

Diante das considerações anteriores perguntase: Os enfermeiros vivenciam sentimentos de insatisfação no labor? Que fatores podem provocar sentimentos de insatisfação no trabalho?

Responder a estas indagações é imprescindível, mostrará aos gestores das instituições hospitalares, que a insatisfação pode afetar à saúde do trabalhador e por consequência prejudicar na qualidade da assistência prestada. Assim sendo, é fundamental garantir condições que favoreçam a satisfação no trabalho do enfermeiro, visto que propicia a promoção da saúde, a prevenção de agravos e consequentemente melhor qualidade de assistencia a população.

Ressalta-se em uma revisão integrativa referente à produção científica brasileira sobre a satisfação e insatisfação da equipe de enfermagem, que mais investigações sobre este assunto devem ser realizadas com métodos análogos e meios de divulgação mais extensos ${ }^{(5)}$.

Este estudo teve como objetivo identificar se os enfermeiros de um Hospital Universitário do Norte do Estado do Paraná vivenciam situações de insatisfação no labor e quais são seus fatores geradores.

\section{MÉTODO}

Pesquisa exploratória, descritiva, com abordagem qualitativa, realizada com enfermeiros de um Hospital de Universitário do interior do Estado do Paraná, mediante autorização da Diretoria de Enfermagem do referido hospital.

Os participantes da pesquisa foram 15 enfermeiros dos turnos matutino, vespertino e noturno das Unidades de Internação de Adultos Masculina e Feminina, Maternidade, Unidade de Terapia Intensiva Adulta, Unidade de Terapia Intensiva de Neonatologia e Pediatria, Pronto Socorro e Unidade de Moléstia Infecciosa , selecionados por intencionalidade e que atenderam aos seguintes critérios de inclusão: ser estatutários, de ambos os sexos, atuar há mais de dois anos na instituição, prestar assistência 
direta aos pacientes nos diferentes setores de atendimento da instituição. Foram excluídos aqueles que ocupavam exclusivamente cargos de chefia e/ou em serviços administrativos e os que se encontravam de férias ou licença.

A definição do número de participantes não se baseou em critérios de representatividade numérica, as entrevistas foram realizadas até o momento em que ocorreu a convergência das falas com relação ao fenômeno estudado ${ }^{(6)}$.

A coleta de dados foi realizada em sala de reunião de um setor de internação do próprio hospital, no período de novembro de 2011 a maio de 2012, por meio entrevistas individuais e gravadas, após assinatura do Termo de Consentimento Livre e Esclarecido.

As entrevistas foram norteadas com roteiro semiestruturado constituído de duas partes: questões objetivas concernentes ao perfil sócio demográfico dos participantes e duas questões norteadoras: Fale-me se você vivência situações de insatisfação no trabalho e que fatores propiciam as vivências de insatisfação.

Para a análise dos resultados utilizouse a técnica de Análise de Conteúdo ${ }^{(7)}$, na modalidade temática com as etapas: leitura, determinação das unidades de registro e significações, codificação e classificação; e o tratamento e interpretação dos resultados obtidos. Através da leitura flutuante, foi possível proceder com as marcações das unidades de registro, organizando-as por temas. Por meio de aproximações e distanciamento construíram-se as categorias e as subcategorias ${ }^{(7)}$.

Para preservar o anonimato os entrevistados foram identificados utilizando-se as letras E1, E2, E3 e, assim, sucessivamente, para a apresentação de seus depoimentos.

O projeto foi aprovado pelo Comitê de Ética em Pesquisa Envolvendo Seres Humanos da Universidade Estadual de Londrina, pelo parecer $\mathrm{n}$. 257 /2011, com CAAE sob o n. 0218.0.268.000-11.

\section{RESULTADOS}

No que concerne ao perfil sócio demográfico, 10 eram mulheres, 5 homens, com idade entre 31 e 58 anos. O tempo de atuação na instituição variou de três a 35 anos. O número de funcionários diretamente subordinados aos enfermeiros variou entre dois e 32.

Das falas dos entrevistados emergiu a categoria vivências de insatisfação no trabalho do enfermeiro com as seguintes subcategorias: a falta de cooperação entre os colegas, a sobrecarga de trabalho, o não reconhecimento do trabalho realizado, a escassez de recursos humanos e materiais e o trabalho em uma instituição pública.

Com relação à subcategoria nominada como a falta de cooperação entre os colegas, esta englobou os discursos que expressam o quanto a falta de cooperação e de solidariedade são fatores que influenciam para as vivências de insatisfação no labor, como demonstram os seguintes fragmentos:

Em relação a alguns colegas eu me sinto insatisfeita, pois não há cooperação, não há ajuda mútua e muitos puxam seu tapete e te empurram contra a parede. (E3)

O que me deixa bastante insatisfeita é que tem colegas que não cooperam, não ajudam e não são solidarias. (E1)

Na subcategoria a sobrecarga no trabalho, a ênfase foi dada à sobrecarga de trabalho do enfermeiro quando necessita exercer funções gerenciais e assistência ao mesmo tempo, conforme apreendido no discurso:

Às vezes tenho que conciliar a gerência com a assistência e, na grande maioria das vezes não da certo aí a gente vive de fato a sobrecarga de trabalho que por sua vez gera insatisfação. (E11)

Os entrevistados na subcategoria denominada o não reconhecimento do trabalho realizado expressaram como fator de vivências de insatisfação quando não são reconhecidos, visto que não se sentem valorizados pelo trabalho realizado, como revelam as falas:

A falta de reconhecimento do nosso trabalho e pelo meu esforço que a gente despende me causa grande insatisfação. (E2)

Às vezes parece que ninguém lhe dá valor, não há reconhecimento. (E7) 
Outra subcategoria desvelada foi a escassez de recursos humanos e materiais. Os enfermeiros afirmaram essa situação como prejudicial para prestar assistência com qualidade, o que se pode verificar nos depoimentos:

Nós enfermeiros quando temos recursos humanos em quantidade inadequada e somos os responsáveis para dar conta da demanda de serviço, para prestar uma assistência com alta qualidade é fator de muita insatisfação. (E1)

A falta de material, de equipamento e trabalhar com recursos humanos em quantidade insuficiente para cuidar do paciente, provoca desmotivação, é muito triste você querer dar o melhor e não ter condições, estes são fatores que causa insatisfação como enfermeira responsável pela assistência. (E13)

No que tange à subcategoria identificada como o trabalho em uma instituição pública, esta ficou evidenciada nos seguintes discursos:

Aquela coisa que todo mundo fala do trabalho em instituição pública que a coisa não anda. Realmente é uma coisa ruim, porque de repente você quer que a coisa ande e a coisa não anda. A resolutividade é demorada e a gente fica insatisfeita, às vezes sinto-me amarrada para resolver as "coisas", mas sei que isso ocorre devido a características de instituição pública. (E11)

O que diminuí minha satisfação é o processo, a burocracia, a demora de conseguir as coisas, ou seja, a gente não tem resolutividade em algumas coisas e eu creio que isso acontece porque trabalhamos em uma instituição pública e tudo depende do governo do Estado, da reitoria e de outros. (E13)

Trabalhar em instituição pública tem-se à impressão que você fala, fala, fala e o seu subordinado está acomodado, claro que são poucos, mas tem. Isto acontece, pois a pessoa sabe que não vai perder o emprego, a posição. (E15)

Denota-se pelas falas a concepção de que a instituição pública "emperra" o desenvolvimento adequado das atividades, sendo esse fato relacionado com a dependência de outras pessoas para que o trabalho tenha resolutividade. Isso provoca sentimentos de insatisfação, já que os enfermeiros sentem-se impotentes diante da burocracia e da acomodação de alguns funcionários.

\section{DISCUSSÃO}

O conhecimento da insatisfação e dos fatores que levam a esse sentimento é de suma importância para os enfermeiros e gerentes das instituições de saúde, visto que propicia a todos uma visão geral das reais necessidades desses trabalhadores. Assim buscam-se ações de ordem coletiva, para diminuir a insatisfação e maximizar a satisfação e, por consequência, resultar em uma melhor assistência aos pacientes, familiares e comunidade.

Para a Psicodinâmica do Trabalho, a cooperação caracteriza-se pelas relações de interdependência dos trabalhadores, em que erros individuais e falhas são diluídos pelo desempenho do grupo, e diferenças individuais e talentos específicos somam-se em prol da qualidade no trabalho. Por meio da cooperação podem-se produzir ações com maior poder de transformação que as realizadas individualmente ${ }^{(8)}$.

Estudo realizado com técnicos de enfermagem de um pronto socorro de um hospital escola de Londrina identificou que bom relacionamento, ajuda mútua e a colaboração foram fatores que levam a união da equipe, convertendo positivamente para a qualidade do trabalho e vivências de prazer $^{(9)}$.

No que diz respeito à sobrecarga foi revelado pelos depoimentos dos participantes do presente estudo, que gera insatisfação no trabalho. A sobrecarga laboral pode levar ao aumento da carga psíquica, que na ausência de vias de descarga satisfatórias, podem transformar-se em tensão e desprazer, levando ao aparecimento de sintomas como astenia e fadiga. Estas consequentemente levarão ao adoecimento, pelo trabalho, que passa ser considerado como fatigante e penoso ${ }^{(10)}$.

Em estudo realizado com enfermeiros em uma instituição hospitalar pública estadual, na cidade de Natal - RN, identificou-se pelas falas dos entrevistados que a sobrecarga de trabalho 
é fonte geradora de sentimentos de insatisfação, de desgaste físico e de estresse ${ }^{(11)}$.

A insatisfação no trabalho e a Burnout tiveram associação estatisticamente significativa em estudo transversal realizado em dois hospitais pediátricos universitários terciários do meio oeste e sul dos Estados Unidos, sobre a qualidade de vida no trabalho e a qualidade do cuidado, ( $n=199$ enfermeiras). A prevalência foi sexo feminino, com média de 8 a 9 anos de trabalho e de 45 anos de idade. Comprovou-se que a insatisfação estava associada à sobrecarga de trabalho relacionada à adequação da equipe ${ }^{(12)}$.

Sabe-se, que sentimentos são difíceis de ser mensurados em estudos quantitativos devido à subjetividade inerente aos mesmos. Assim sendo, é fundamental que estudos sejam realizados por meio de pesquisas qualitativas ${ }^{(12)}$.

Verificou-se também pelos depoimentos dos participantes da presente investigação que ocorre insatisfação por não se sentirem valorizados e reconhecidos por aquilo que realizam. Sabe-se que quando os esforços para a realização do trabalho não são valorizados ou passam despercebidos por aqueles que participam do processo, existe o sofrimento e, consequentemente, os perigos que ele oferece à saúde do trabalhador ${ }^{(13)}$.

O reconhecimento é fator necessário para a satisfação do trabalhador e, como fonte de prazer pela valorização profissional, expressa a sua influência na saúde do indivíduo, bem como na qualidade do trabalho realizado(13-14).

Em um estudo desenvolvido com profissionais de enfermagem de um Hospital Geral de Jacarepaguá e de um Hospital Público de médio porte, da cidade do Rio de Janeiro, identificou que a falta de reconhecimento foi um fator de importância para a insatisfação dos profissionais entrevistados $^{(15)}$.

Em outro estudo desenvolvido com enfermeiros de instituições públicas e privadas da cidade de Concepcion, Chile, com o objetivo de identificar a satisfação e insatisfação no labor, demonstrou que a falta de reconhecimento pelo trabalho realizado foi um dos fatores geradores de sentimentos de insatisfação no trabalho dos profissionais $^{(16)}$.

Em um estudo transversal realizado no Japão com 5.956 enfermeiras identificou as situações de Burnout, insatisfação no trabalho e qualidade de cuidado, determinando que esses fatores estavam relacionados ao ambiente de trabalho em 19 hospitais japoneses (15 desses hospitais universitários). Entre as enfermeiras, 56\% apresentavam exaustão emocional, 60\% relataram insatisfação no trabalho e 59\% relataram que a qualidade de cuidado nos hospitais era ruim ou precária. Tais resultados sugerem que essas condições estão associadas ao ambiente de trabalho e a uma ampla porcentagem de enfermeiras inexperientes ${ }^{(17)}$.

Concluiu-se que uma equipe inadequada ou inexperiente para proporcionar os cuidados, e lidar com problemas de relacionamento e cooperação entre os médicos e enfermeiros está significativamente associada à Síndrome de Burnout e os sentimentos de insatisfação no trabalho ${ }^{(17)}$.

O processo de trabalho do enfermeiro enquanto líder propicia o sofrimento psíquico, devido às situações de angústia e estresse que vivencia em decorrência desta responsabilidade ${ }^{(18)}$.

Em um estudo que teve como objetivo conhecer as percepções dos trabalhadores da enfermagem acerca da satisfação ou insatisfação no contexto do trabalho da Unidade de Terapia Intensiva revelou que a insuficiência de recursos materiais foi justificativa apresentada pelos trabalhadores para mostrarem-se insatisfeitos com sua atividade laboral ${ }^{(19)}$.

A frustração em relação à falta de materiais vivenciada pelos trabalhadores leva-os à realização constante de improvisações para o desenvolvimento das atividades assistenciais, deixando-os insatisfeitos e sujeitos ao sofrimento. Ao avaliar a repercussão das condições de trabalho sob o enfoque do sofrimento entre profissionais de enfermagem de um serviço público, observouse que as condições de trabalho foram descritas como determinantes de sofrimento devido à falta de materiais que comprometiam a qualidade da assistência prestada ${ }^{(20)}$.

Sabe-se que há discussões voltadas ao aumento da eficiência e qualidade dos serviços públicos de saúde que apontam, entre outras ações, a preocupação em relação à gestão de recursos humanos, em que os mesmos devem-se organizar objetivando as necessidades funcionais da instituição ${ }^{(21)}$.

Há anos é constante o baixo investimento nos setores de saúde no Brasil, refletindo na 
qualidade dos serviços, nos baixos salários, na falta de reposição dos recursos pessoais, levando a diferentes modalidades de contratação, a fim de suprir estas necessidades, e a dificuldades de gerenciamento nestes setores ${ }^{(22)}$.

Ao comparar as condições de trabalho dos profissionais de saúde no setor público em decorrência dos investimentos realizados pelos órgãos governamentais, um estudo(23) apontou que a satisfação destes profissionais foi menor quando comparada a trabalhadores em instituições hospitalares particulares e beneficentes. Observou-se que nos hospitais estaduais encontrava-se o maior nível de insatisfação em relação à remuneração, benefícios e até desrespeito com os profissionais.

Verifica-se ainda que em instituições públicas, a autonomia de gestão dos recursos humanos é bem limitada, dificultando a contratação e demissão de funcionários que não querem produzir. Além disso, alguns trabalhadores, cientes da quase impossibilidade de demissões devido à estabilidade que o setor público lhes oferece, deixam de executar suas atividades e até desafiam seus superiores ${ }^{(21)}$.

Quanto à resolutividade nos serviços, os resultados desta pesquisa são semelhantes a um estudo que teve por objetivo identificar as situações geradoras de prazer e de sofrimento no trabalho entre Agentes Comunitários. Este identificou que as deficiências nos serviços de saúde são fatores que causam sofrimento no trabalho, como, por exemplo, a falta de resolutividade dos serviços ${ }^{(24)}$.

Ser resolutivo leva à possibilidade de materialização do trabalho, no qual o trabalhador consegue dar sentido ao esforço dispensado para a realização da atividade, o que pode se constituir como uma estratégia para evitar a insatisfação no labor, ou seja, propiciar vivências de satisfação ${ }^{(24)}$.

\section{CONSIDERAÇÕES FINAIS}

Ao final do estudo pode-se verificar que as vivências de insatisfação no trabalho dos enfermeiros originaram-se de fatores de ordem subjetiva como: a falta de cooperação entre os colegas, a sobrecarga de trabalho e o não reconhecimento do trabalho e, por fatores relacionados diretamente com a instituição como demonstrado pelas falas, como a escassez de recursos humanos, materiais e o trabalho no setor público.

Destaca-se que os objetivos do estudo foram alcançados, porém apresentou limitações devido a insatisfação no trabalho é objeto de investigação que envolve o abstrato, a subjetividade, ou seja, não é tido como um "mundo concreto" e sofre influências pelo tempo, pelo espaço e pelas características individuais de cada enfermeiro. Assim, torna-se necessário considerar os resultados em sua singularidade, visto que um dos limites impostos pelo método empregado é não permitir a generalização dos mesmos.

Porém, acredita-se que o conhecimento revelado poderá suscitar discussões e reflexões sobre o processo de trabalho e, assim, fomentar a busca por meio de ações coletivas, de estratégias para minimizá-las e ou resolvê-las, propiciando a promoção da saúde, a prevenção de doenças e a diminuição dos agravos à saúde destes profissionais. Pode se afirmar que o estudo contribuiu com evidências que podem subsidiar novas pesquisas voltadas para a saúde do trabalhador no que tange a satisfação no trabalho.

Por fim, frente aos resultados desvelados nesta pesquisa sugere-se se ainda que as escolas de enfermagem, tanto na graduação como pósgraduação, reflitam sobre a necessidade de inserir em seus currículos, ou conteúdos programáticos, temas, módulos, disciplinas, dentre outros, que abordem a Saúde do Trabalhador.

\section{REFERÊNCIAS}

1. Silva RM, Beck CLC, Guido LA, Lopes LFD, Santos JLG. Análise quantitativa da satisfação profissional dos enfermeiros que atuam no período noturno. Texto Contexto Enferm. [Internet] 2009;18(2) [acesso em 10 out 2012]. Disponível: http://dx.doi.org/10.1590/ S0104-07072009000200013.

2. Nunes CM, Tronchin DMR, Melleiro MM, Kurcgant P. Satisfação e insatisfação no trabalho na percepção de enfermeiros de um hospital universitário Rev. Eletr. Enf. [Internet] 2010;12(2) [acesso em 05 out 2012]. Disponível: http://www.fen.ufg.br/revista/v12/n2/ v12n2a04.htm.

3. Dejours C. A loucura do trabalho: estudo de psicopatologia do trabalho. $5^{\mathrm{a}}$ ed. São Paulo: Cortez; 1992.

4. Angeli AAC, Luvizaro NA, Galheigo SM. O cotidiano, o lúdico e as redes relacionais: a artesania do cuidar 
em terapia ocupacional no hospital. Interface [Internet] 2012;16(40) [acesso em 13 jul 2012]. Disponível: http://dx.doi.org/10.1590/S1414-32832012005000016

5. Melo MB, Barbosa MA, Souza PR. Satisfação no trabalho da equipe de enfermagem: revisão integrativa. Rev. Latino-Am. Enfermagem. [Internet] 2011;19(4) [acesso em 26 dez 2012]. Disponivel: http://dx.doi. org/10.1590/S0104-11692011000400026

6. Minayo MCS. O desafio do conhecimento: pesquisa qualitativa em saúde. São Paulo: Hucitec; 2010.

7. Bardin L. Análise de conteúdo. Lisboa: Edições 70; 2011.

8. Mendes AM. Psicodinâmica do trabalho: teoria, método e pesquisas. São Paulo: Casa do Psicólogo; 2007.

9. Garcia $A B$, Dellaroza MSG, Haddad MCL, Pachemshy LR. Prazer no trabalho de técnicos de enfermagem do pronto-socorro de um hospital universitário público. Rev. Gaúcha Enferm. [Internet] 2012;33(2) [acesso em 06 nov 2012]. Disponível: http://dx.doi.org/10.1590/ S1983-14472012000200022

10. Dejours C, Abdoucheli E, Jayet C. Psicodinamica do trabalho: contribuição da escola dejouriana a analise da relação prazer, sofrimento e trabalho. São Paulo: Atlas; 2011.

11. Fernandes SMBA, Medeiros SM, Ribeiro LM. Estresse ocupacional e o mundo do trabalho atual: repercussões na vida cotidiana das enfermeiras. Rev. Eletr. Enf. [Internet] 2008;10(2) [acesso em 03 jul 2012]. Disponível: http://www.fen.ufg.br/revista/v10/ n2/v10n2a13.htm

12. Holden RJ, Scanlon MC, Patel NR, Kaushal $\mathrm{R}$, Escoto $\mathrm{KH}$, Brown RL, et al. A human factors framework and study of the effect of nursing workload on patient safety and employee quality of working life. BMJ Qual Saf. 2011; 20(1):15-24.

13. Dejours C. A banalização da injustiça social. Rio de Janeiro: FGV; 2000.

14. Prestes FC, Beck CLC, Silva RM, Tavares JP, Camponogara S, Burg G. Prazer-sofrimento dos trabalhadores de enfermagem de um serviço de hemodiálise. Rev. Gaúcha Enferm. [Internet] 2010;31(4) [acesso em 25 jul 2012]. Disponível: http://dx.doi. org/10.1590/S1983-14472010000400018

15. Hanzelman RS, Pasos JP. Imagens e representações da enfermagem acerca do stress e sua influência na atividade laboral. Rev Esc Enferm USP. [Internet] 2010;44(3) [acesso em 30 ago 2012] Disponível: http:// dx.doi.org/10.1590/S0080-62342010000300020

16. Fernandez Larraguibel B, Paravic Klinjn T. Nível de satisfação laboral em enfermeras de hospitales publicos y privados de la provincia de Concepcion, Chile. Cienc. Enferm. 2003;9(2):57-66.
17. Kanai-Pak M, Aiken LH, Sloane DM, Poghosyan L. Poor work environments and nurse inexperience are associated with burnout, job dissatisfaction, and quality deficits in Japanese hospitals. J. Clin. Nurs. [Internet] 2008;17(24) [acesso em 20 mar 2013]. Disponível: http://onlinelibrary.wiley.com/doi/10.1111/ j.1365-2702.2008.02639.x/pdf

18. Gobbi C, Durman S. Sofrimento psíquico no trabalho: percepções de enfermeiros. Rev. Tempus Actas Saúde Colet. [Internet]. 2010;4(1) [acesso em 21 abr 2012]. Disponível: http://www.tempusactas.unb.br/index. php/tempus/article/viewFile/953/898.

19. Silveira RS, Funck CR, Lunardi VL, Silveira JT, Avila LI, Lunardi Filho WD, et al. Percepção dos trabalhadores de enfermagem acerca da satisfação no contexto do trabalho na UTI. Enferm. Foco [Internet] 2012;3(2) [acesso em 20 out 2012]. Disponível: http://revista. portalcofen.gov.br/index.php/enfermagem/article/ view/262

20. Medeiros SM, Ribeiro LM, Fernandes SMBA, Veras VSD. Condições de trabalho e enfermagem: a transversalidade do sofrimento no cotidiano. Rev. Eletr. Enferm. [Internet] 2006;8(2) [acesso em 4 ago 2012]. Disponível: http://www.fen.ufg.br/revista/revista8_2/ v8n2a08.htm.

21. Farias LO, Vaitsman J. Interação e conflito entre categorias profissionais em organizações hospitalares públicas. Cad. Saúde Pública. [Internet] 2002;18(5) [acesso em 26 set 2012]. Disponível em: http://www. scielo.br/pdf/csp/v18n5/10995.pdf.

22. Rebouças D, Legay LF, Abelha L. Satisfação com o trabalho e impacto causado nos profissionais de serviço de saúde mental. Rev Saúde Pública. [Internet] 2007;41(2) [acesso em 26 set 2012]. Disponível: http:// www.scielo.br/pdf/rsp/v41n2/5992.pdf.

23. Lima Júnior J, Alchiri JC, Maia EMC. Avaliação das condições de trabalho em Hospitais de Natal, Rio Grande do Norte, Brasil. Rev EscEnferm USP [Internet]. 2009;43(3) [acesso em 25 nov 2012]. Disponível: http:// www.scielo.br/pdf/reeusp/v43n3/a24v43n3.pdf.

24. Lopes DMQ. Agentes comunitários de saúde e as vivências de prazer - sofrimento no trabalho: estudo qualitativo. Rev Esc Enferm USP [Internet] 2012;46(3) [acesso em 25 nov 2012]. Disponível: http://www. scielo.br/scielo.php?script=sci_arttext\&pid=S008062342012000300015\&lng=pt\&nrm=iso 